\title{
Relações entre instituições, capital humano e acumulação de capital físico nos municípios brasileiros
}

\author{
Gilberto da Silveira Barros Neto* \\ Luciano Nakabashi*
}

\begin{abstract}
RESUMO - O objetivo do presente estudo é analisar as inter-relações entre acumulação de capital físico, capital humano e instituições nos municípios brasileiros entre 1991 e 2000, com ênfase no impacto da variação destes sobre a mudança do primeiro. A análise empírica indica que elevados níveis de capital humano per capita estão associados a uma rápida expansão do capital físico per capita no decorrer da década em análise. A proxy para instituições também é positiva e significativa na maior parte das especificações, mesmo quando se controla para o problema da endogeneidade. Variáveis dummies estaduais sugerem efeitos regionais significativos.
\end{abstract}

Palavras-chave: Capital físico. Capital humano. Instituições.

\section{INTRODUÇÃO}

O presente artigo não procura sugerir que a acumulação de capital físico seja a questão central no desenvolvimento econômico. Mesmo Solow (1957) e, recentemente, Easterly e Levine (2001) trazem evidências que este não é o caso em exercícios de contabilidade de crescimento. Hall e Jones (1999) encontraram uma diferença entre produto per capita dos 5 países mais ricos e os 5 mais pobres de, aproximadamente, 32 vezes. Decomposta entre intensidade de capital, capital humano e PTF, o primeiro respondia por um fator de 1,8; o segundo por 2,2; e o último por $8,3^{1}$.

No entanto, explorar as interações existentes entre a acumulação de capital físico, capital humano e instituições ajuda a entender o processo de crescimento econômico de uma forma geral. Nosso argumento é que considerar o papel separado de cada fator de produção na determinação do produto não revela a total relevância dos mesmos nesse processo. Por exemplo, Lucas (1990) enfatiza que uma das razões que restringe o fluxo de capital para os países menos desenvolvidos é a sua complementariedade com o fator capital humano. Adicionalmente, esse fator de produção pode ter um papel mais relevante em determinadas situações. Por exemplo, o próprio caso brasileiro, constatado por Easterly e Levine (2001), mostra uma trajetória distinta em relação à regularidade mundial: no período 1940-80 o crescimento médio anual do produto

*Mestrando em Desenvolvimento Econômico pela Universidade Federal do Paraná. Endereço eletrônico: gilsbn@ gmail.com.

** Doutor em Economia pela Universidade Federal de Minas Gerais. É professor do Departamento de Economia da Universidade Federal do Paraná e pesquisador do CNPq. Endereço eletrônico: luciano.nakabashi@gmail.com.

1 Dados de 1988. 
alcançou 6,4\%, sendo a proporção da contribuição do capital físico, trabalho e PTF de 51\%, $20 \%$ e $29 \%$, respectivamente ${ }^{2}$. Ou seja, no segundo e terceiro quartos do século XX o país apresentou um crescimento baseado em aprofundamento de capital - mesmo padrão da América Latina e leste da Ásia ${ }^{3}$. O Gráfico 1 mostra a relação entre a proxy do capital físico per capita ${ }^{4}$ o PIB per capita entre os municípios brasileiros para o ano de $2000^{5}$.

GRÁFICO 1 - RELAÇÃO ENTRE O PIB PER CAPITA E O CAPITAL FÍSICO PER CAPITA NOS MUNICÍPIOS BRASILEIROS

FONTE: Ipeadata.

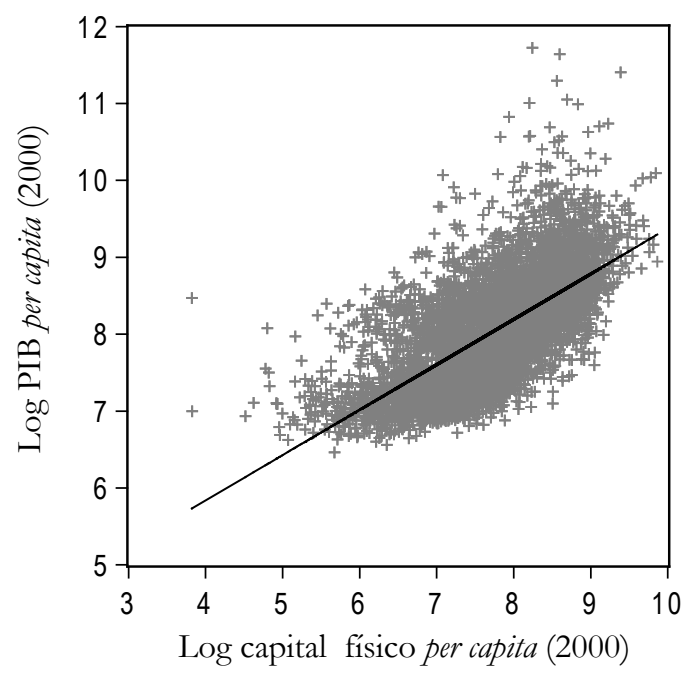

Existem estudos empíricos que analisam a dinâmica do investimento privado no Brasil, mas a quase totalidade deles utiliza séries temporais e busca impactos no investimento agregado das seguintes variáveis explicativas: (i) variação da demanda agregada (produto ou utilização da capacidade instalada); (ii) crédito (juros e nível de crédito); (iii) preços dos fatores; (iv) investimento público ${ }^{6}$; (v) instabilidade econômica (inflação, câmbio etc.); condições externas (dívida externa etc.). É fácil verificar que muitas das variáveis são insignificantes ou constantes entre os municípios, sendo as diferenças de investimento líquido entre as municipalidades independentes desse tipo de variável ${ }^{7}$.

2 As melhoras no capital humano estão embutidas na PTF.

3 Os dados são da Tabela 1 de Easterly e Levine (2001). Os países da América Latina listados são: Argentina, Brasil, Chile, México e Venezuela. Destes, somente o Chile obteve uma participação da PTF no crescimento superior ao da acumulação de capital. Da Ásia, os países listados foram: Hong Kong, Singapura, Coreia do Sul e Taiwan. Em nenhum dos países desse grupo a PTF assumiu posição principal.

4 A Seção 3 traz a definição da proxy do capital físico.

5 Coeficiente angular de 0,59 e $\mathrm{R}^{2}=0,46$.

6 Analisa o efeito dominante sobre investimento privado do investimento público: crowding out ou crowding in.

7 Ver Melo e Rodrigues Júnior (1998); Alves e Luporini (2007) e Sonaglio, Braga e Campos (2010). O segundo trás uma tabela com o resumo dos principais estudos empíricos que envolvem o Brasil, destacando as variáveis dependentes, as explicativas e os sinais dos coeficientes em cada estudo. 
No entanto, esses estudos não consideram as relações existentes entre capital físico e humano além dos efeitos das instituições sobre os investimentos. Visando preencher essa lacuna, o objetivo do presente artigo é analisar os impactos das instituições e do capital humano sobre o investimento líquido entre os municípios brasileiros na década de 1990. Barro (1991) e Benhabib e Spiegel (1994) trouxeram evidências indicando a importância dessas variáveis em análises cross-country.

As conclusões básicas apontam para uma associação positiva entre o nível de capital humano inicial (1991) e posterior acumulação de capital (1991-2000). Efeitos regionais, captados pelas dummies estaduais, exercem influência significativa sobre a acumulação. Entende-se que os efeitos dos estados refletem estrutura de impostos, além de políticas regionais. As instituições municipais também são relevantes para o crescimento no nível de capital físico per capita municipal.

Além dessa introdução, apresentamos uma breve revisão teórica em seguida. Na Seção 3 apresentamos os dados utilizados na análise empírica e na Seção 4 os resultados das regressões econométricas e a interpretação dos mesmos. Por fim, as conclusões.

\section{TEORIA: DETERMINANTES DO INVESTIMENTO}

Nos modelos de crescimento endógeno, Lucas (1988) coloca o capital humano com spillovers que aumentam a produtividade do capital físico e do trabalho. Romer (1990) criou uma função de progresso técnico, onde a quantidade de capital humano dedicado a P\&D define a taxa do avanço tecnológico e, consequentemente, do produto per capita ${ }^{8}$. A importância do capital humano também se dá através da difusão tecnológica, como apontam os trabalhos empíricos de Benhabib e Spiegel (1994) e Nakabashi e Figueiredo (2008); ambos baseados no modelo de difusão proposto por Nelson e Phelps (1966). O capital humano acelera o fechamento do gap tecnológico?.

Os spillovers de Lucas (1988), ao aumentarem a produtividade marginal do capital físico, induzem seu acúmulo: economias com alto capital humano tenderão a drenar para si o

8 Os modelos endógenos não passam sem críticas: Jones (1995a) mostra que o nível de capital humano dedicado à pesquisa, pelo menos para os países da OCDE, aumentou exponencialmente desde a década de 1950, sem que com isso fossem verificados aumentos nas taxas de crescimento de longo prazo. Jones (1995b) propôs um modelo semiendógeno onde a taxa de crescimento do estoque de capital humano dedicado à pesquisa - e não mais o seu nível - é que determina a taxa de crescimento. Tais críticas são importantes, mas não mudam o modo como o capital humano influencia a produtividade do capital físico.

9 A taxa de crescimento do produto per capita é proporcional ao gap. Diferenças no nível de capital humano podem ser interpretadas como um fator que multiplica o hiato, alterando a velocidade de convergência entre os países, que passa a ser função da intensidade do capital humano. Nakabashi e Figueiredo vão mais além, identificando impactos indiretos através da interação do capital humano também com importações e investimento externo direto. 
capital físico, o que está de acordo com a evidência empírica. Nos modelos de difusão, a lógica é um pouco mais complexa. Um alto nível de capital humano levaria a um alto nível tecnológico ou uma rápida incorporação de tecnologia. Em ambos os casos é de se esperar uma alta produtividade marginal do capital físico. Lógica semelhante se aplica aos modelos endógenos do tipo Romer (1990). O Gráfico 2 mostra a relação positiva entre o capital fixo e capital humano nos municípios brasileiros ${ }^{10}$. Portanto, o capital humano pode elevar a produtividade marginal do capital físico tanto diretamente, pela complementariedade entre eles, quanto indiretamente, pelos seus efeitos no progresso tecnológico. Em todos esses modelos, o acúmulo do capital físico, ceteris paribus, reduz sua produtividade marginal. Desse modo, o investimento líquido em capital físico deve ser menor quanto maior a quantidade do mesmo, no período inicial, em relação aos outros insumos.

GRAFICO 2 - RELAÇÃO ENTRE O CAPITAL FÍSICO PER CAPITA E CAPITAL HUMANO PER CAPITA NOS MUNICÍPIOS BRASILEIROS

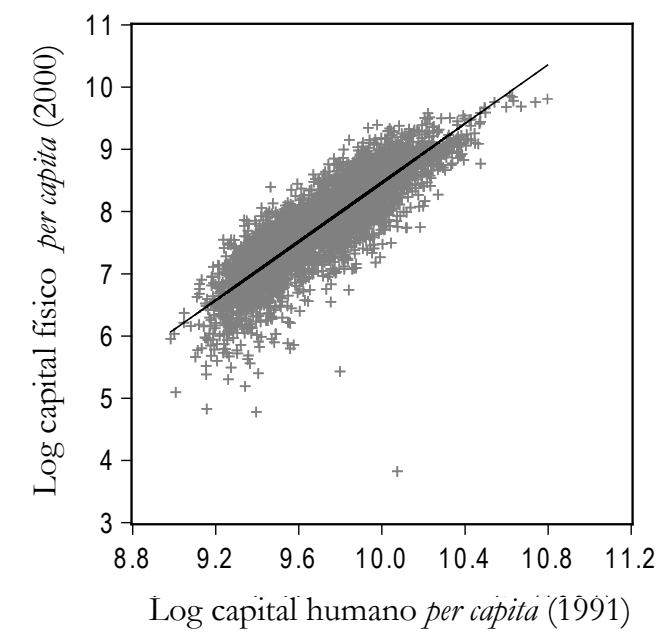

FONTE: Ipeadata.

Em relação às instituições, Sala-i-Martin (2002) faz a seguinte divisão: leis (direito de propriedade, força da lei, paz); funcionamento dos mercados (estruturas dos mercados, políticas de competição, abertura para os mercados externos, capital e tecnologia); instituições políticas (democracia, liberdade política, ruptura política, estabilidade política); sistema de saúde; mercado financeiro (sistema bancário e de ações); instituições governamentais (tamanho da burocracia, red tape $e^{11}$, corrupção do governo) e desigualdade e outros conflitos sociais. Portanto, elas afetam diretamente os investimentos de acordo com o grau de respeito à propriedade privada, desenvolvimento do mercado financeiro, tamanho da burocracia, funcionamento dos 10 Coeficiente de correlação de 3,01 e $\mathrm{R}^{2}=0,72$.

11 Regulações e processos burocráticos que impedem ou dificultam a tomada de decisão por parte dos agentes econômicos. Quanto maior a red tape, maior o espaço para corrupção e rent seeking, na medida em que os agentes tentam acelerar o processo burocrático ou obter rendas através de privilégios da burocracia. 
mercados etc.

Adicionalmente, as instituições afetam os processos de difusão e inovação de tecnologia, além da acumulação do capital humano, afetando de forma indireta a acumulação de capital físico (HALL; JONES, 1999). Por exemplo, a falta de difusão da tecnologia existente seria apenas o reflexo de instituições incapazes de fornecer os incentivos necessários para sua propagação. A grande variância de produto per capita e produtividade evidenciada nas análises cross-country teria raízes no diferencial das instituições formadas ao longo da história dos países.

Acemoglu, Johnson e Robinson (AJR) $(2001 ; 2002)$ defendem que as melhores instituições iniciais - ao dar mais incentivos para acumulação de fatores e desenvolvimento e difusão de novas ideias - permitiram que países como EUA e Canadá se aproveitassem largamente da revolução industrial, decolando, em relação ao resto da América, a partir da segunda metade do século XVIII ${ }^{12}$. Engerman e Sokoloff (2002) produzem um argumento complementar, a saber, que as instituições implantadas nas colônias americanas seguiram um padrão de acordo com as dotações iniciais: algumas partes do continente eram propícias a produção de produtos com alto valor no mercado europeu (e.g., açúcar, tabaco etc.), com uma produção caracterizada por grandes latifúndios e trabalho escravo. Tal estrutura facilitou a formação de sociedades altamente desiguais, polarizadas entre uma reduzida elite local e uma vasta população de escravos ou homens livres pobres, com escassos direitos de propriedade. Essa conjuntura permitiu a criação e manutenção de instituições que beneficiassem esta elite em detrimento de grande parte da população.

Easterly e Levine (2002) e Acemoglu et al. (2003) trazem evidências, inclusive, da dominância das instituições sobre políticas macroeconômicas: quando controlada pelas instituições, políticas macroeconômicas não têm poder de previsão do desempenho econômico de longo prazo. São apenas reflexos da realidade institucional, do conflito de interesse e rent seeking ${ }^{13}$.

Naritomi (2007) e Pereira, Nakabashi e Sachsida (2010) encontraram uma correlação

12 Segundo AJR, no caso das ex-colônias europeias, as instituições iniciais seriam melhores (com direitos de propriedade bem definidos e restrições ao Executivo) quanto maior a proporção de europeus, em relação a população total, assentados nas colônias. Onde se estabeleceram em grandes números, os europeus (e descendentes) tenderiam a exigir instituições similares àquelas dos seus países de origem. $\mathrm{Na}$ situação inversa, a metrópole estabelecia "colônias de exploração", com direitos de propriedade restritos às elites. A proporção da população de origem europeia nas colônias pode ser explicada a partir de sua "taxa potencial de mortalidade", registrada nas campanhas militares das potências europeias e missões religiosas (principalmente na América) patrocinadas pelo Vaticano. AJR (2001; 2002) instrumentalizaram as instituições (as quais tinham como proxies a restrição ao Executivo no início do século XX; índice democrático etc.) através da "mortalidade potencial” (MQ2E), encontrando coeficientes significativos para instituições - variável explicativa para o desenvolvimento econômico atual.

13 Através da mera substituição de políticas macroeconômicas equivocadas (e.g., grande consumo do governo, inflação e valorização real excessiva do câmbio) muda-se apenas a forma de extração de rendas pelo grupo no poder: as políticas refletem sintomas institucionais, e não causas do subdesenvolvimento. 
positiva entre o produto per capita dos municípios brasileiros e instituições, instrumentalizando-as através de variáveis históricas e geográficas, respectivamente. Os primeiros construíram índices da influência do ciclo do ouro e da cana-de-açúcar: quanto mais próximos desses ciclos, piores as instituições municipais, seja por concentração de terra e poder político, por herança de administrações públicas repressivas e ineficientes ou, ainda, falta de acesso à justiça. No segundo, as instituições foram instrumentalizadas através de variáveis geográficas que serão utilizadas também neste trabalho. É válida a constatação que a área de influência dos ciclos da cana-de- açúcar e do ouro guarda alguma relação com a distância do Equador.

Neste trabalho, procura-se mensurar a importância do que Naritomi (2007) classifica como "microinstituições", conceitualmente diferenciadas das "macroinstituições" - estas ligadas aos direitos de propriedade, herança legal etc.

\section{DADOS}

Da seção anterior identificam-se as variáveis que influenciam a acumulação de capital físico per capita: (i) nível do capital físico per capita inicial (-); (ii) nível do capital humano per capita inicial (+); (iii) qualidade das instituições $(+)$. $\mathrm{Na}$ análise empírica, o custo de capital pode ser incluído na constante da regressão ${ }^{14}$.

As variáveis necessárias para o exame empírico proposto estão disponíveis em frequência decenal, construídas com base em Censos. Serão utilizados dados de 1991 e 2000. A variável dependente é a proxy do estoque de capital físico per capita final dividida pela mesma variável no período inicial $\left(D K_{p c}\right)^{15}$; os regressores: (i) estoque de capital físico per capita $\left(K_{p c}\right)$; (ii) estoque de capital humano per capita $\left(H_{p c}\right)^{16}$; (iii) índice de desigualdade de Theil ${ }^{17}$. Existem dois motivos para inserir o índice de desigualdade como regressor. Primeiramente, Sala-i-Martin (2002) coloca as desigualdades sociais como fazendo parte do vetor institucional global. Adicionalmente, pode-se testar se a desigualdade, per se, interfere na expansão do capital físico. Por último, (iv) o Índice de Qualidade Institucional Municipal (IQIM) criado pelo Ministério do Planejamento e

14 Espera-se que os custos de capital (depreciação, juros e taxação sobre os ganhos) não variem entre os municípios.

15 Série denominada "capital residencial urbano" no Ipeadata. Consiste no fluxo perpétuo (constante) de aluguéis mensais descontados à taxa de desconto de $0,75 \%$ a.m.

16 Série denominada "capital humano" no Ipeadata. Dado o valor esperado presente dos rendimentos anuais (descontados a 10\% a.a.) associados à escolaridade e experiência (idade) da população em idade ativa (15 a 65 anos), o estoque de capital humano é calculado pela diferença entre o rendimento obtido no mercado de trabalho e a estimativa daquele obtido por um trabalhador sem escolaridade e experiência.

17 Desigualdade na distribuição dos indivíduos segundo a renda domiciliar per capita. É definido como logaritmo da razão entre as médias aritmética e geométrica das rendas individuais (sendo nulo quando não houver desigualdade de renda entre os indivíduos e tendendo ao infinito quando a desigualdade tender ao máximo) Do Ipeadata. 
Gestão e fornecido por Pereira, Nakabashi e Sachsida (2010), está disponível apenas para o ano de 2000. Com base no pressuposto da inércia institucional, utilizá-lo como regressor não prejudica a análise. Uma vez que (iii) e (iv) guardam baixa correlação entre si, entende-se que esses dois aspectos são relativamente independentes e medem características institucionais distintas.

A projeção linear de $D K_{p c}$ contra $H_{p c}$ IQIM e demais variáveis explicativas (MQO), traz a expectativa de endogeneidade entre a acumulação de capital físico, capital humano e instituições. Adicionalmente, existem variáveis omitidas que podem estar correlacionadas com os regressores. Para contornar tais problemas, o uso de variáveis instrumentais é suficiente. Serão utilizados quatro instrumentos, os quais servirão tanto para IQIM quanto para o capital humano: latitude; precipitação anual; temperatura média anual; e altitude do município ${ }^{18}$. Tais variáveis geográficas podem ter influenciado a colonização de acordo com a Seção 2 deste artigo. Easterly e Levine (2002) e Hall e Jones (1999) utilizaram variáveis semelhantes como instrumentos em análises cross-country; Pereira, Nakabashi e Sachsida (2010) utilizam essas mesmas variáveis (e fracionamento étnico); Naritomi (2007) utiliza essas e outras variáveis geográficas como instrumentos para instituições municipais. O processo de Mínimos Quadrados em dois Estágios (MQ2E), através dos instrumentos, busca mensurar os impactos de variações exógenas de $H_{p c}$ e IQIM em $D K_{p c}$.

\section{RESULTADOS}

A Tabela 1 apresenta os resultados do MQO. As variáveis $D K_{p c}, K_{p c}$ e $H_{p c}$ foram transformadas em logaritmos, portanto, seus coeficientes medem elasticidades. O capital físico, humano e as instituições são significativos (a 1\%) em todos os casos. O sinal de $K_{p c}$ indica que a produtividade marginal do capital, quando controlada por outras variáveis, é decrescente: níveis elevados de $K_{p c}$ estão associados com menor acumulação futura. O sinal positivo do capital humano está de acordo com a descrição dos modelos endógenos, indicando que quanto maior a intensidade desse fator no período inicial, maior a produtividade marginal (e acumulação) do capital físico. A qualidade institucional tem efeito positivo sobre $D K_{p c}$, dado o sinal positivo do IQIM e negativo do índice de Theil.

No modelo (2), a inclusão do IQIM implica uma redução significativa do impacto de $H_{p c}$ (cerca de 16\%) sobre $D K_{p c}$. Resultado esperado, dado que o modelo teórico incluía instituições como variável explicativa. Em (3), o índice de desigualdade de Theil tem efeito negativo e significativo, e pouco altera a magnitude dos regressores em relação ao modelo (2).

18 Assim como em Glaeser et al. (2004), neste caso tanto o capital humano quanto as instituições serão instrumentalizados pelas variáveis geográficas: segundo os autores, o choque exógeno da colonização europeia afetou não só o processo histórico através das instituições, como também do estoque de capital humano inicial. 
TABELA 1 - ACUMULAÇÃO DE CAPITAL FÍSICO PER CAPITA (1991-2000)

\begin{tabular}{|c|c|c|c|c|c|}
\hline & (1) & (2) & (3) & (4) & (5) \\
\hline \multicolumn{6}{|c|}{ Variável dependente: $\log _{e} D K_{t c}=\log _{e}\left[K_{t c(2000)} / K_{t c(1991)}\right]$} \\
\hline Const. & -2.3418 & -1.6836 & -1.7552 & -0.0568 & -0.1694 \\
\hline Const. & $(0.31986)^{* * *}$ & $(0.34194)^{* * *}$ & $(0.34784)^{* * *}$ & $(0.43070)$ & $(0.43010)$ \\
\hline $\log _{\rho} K_{p c}$ & -0.3686 & -0.3652 & -0.3682 & -0.3575 & -0.3596 \\
\hline$(1991)^{p c}$ & $(0.01489)^{* * *}$ & $(0.01482)^{* * *}$ & $(0.0151)^{* * *}$ & $(0.0168)^{* * *}$ & $(0.01679)^{* * *}$ \\
\hline $\log H$ & 0.5798 & 0.4859 & 0.5000 & 0.3334 & 0.3331 \\
\hline$(1991)^{p c}$ & $(0.04324)^{* * *}$ & $(0.04658)^{* * *}$ & $(0.04774)^{* * *}$ & $(0.05627)^{* * *}$ & $(0.0562) * * *$ \\
\hline IOIM & & 0.0767 & 0.0781 & 0.0333 & 0.0331 \\
\hline$(2000)$ & & $(0.00852)^{* * *}$ & $(0.00853)^{* * *}$ & $(0.00809)^{* * *}$ & $(0.00806)^{* * *}$ \\
\hline Theil & & & -0.0976 & -0.1060 & -0.1087 \\
\hline$(1991)$ & & & $(0.02888)^{* * *}$ & $(0.02786)^{* * *}$ & $(0.02784)^{* * *}$ \\
\hline $\begin{array}{l}\text { Dum. Est. } \\
\text { (valor-p) }\end{array}$ & & & & $(0.00 \mathrm{e}+00)^{* * *}$ & $(0.00 \mathrm{e}+00)^{* * *}$ \\
\hline Latitude & & & & & 0.0061 \\
\hline Hausman & $H a c$ & Htc IOIM & Hts IOIM & & (0) \\
\hline & 0.455 & $\begin{aligned} \Pi p c, 18 \\
0.466\end{aligned}$ & $\begin{aligned} 110 c, 18 \\
0.468\end{aligned}$ & 0.551 & 0.551 \\
\hline $\mathrm{R}^{2}$ ajust. & 0.454 & 0.466 & 0.467 & 0.548 & 0.548 \\
\hline $\mathrm{n}$ & 4491 & 4489 & 4489 & 4489 & 4489 \\
\hline
\end{tabular}

NOTA: Erros-padrão robustos; ${ }^{* * *}$ significativo a $1 \%$.

O nível do IQIM não foi transformado em logaritmo; seu coeficiente é uma semielasticidade. Como mostra a parte final da Tabela 1, através do teste de Hausman, os regressores $H_{p c}$ e IQIM são endógenos. A Tabela 2 apresenta o segundo estágio do MQ2E quando tanto $H_{p c}$ e IQIM são instrumentalizados pelas variáveis geográficas ${ }^{19}$.

TABELA 2 - ACUMULAÇÃO DE CAPITAL (1991-2000) - SEGUNDO ESTÁGIO DO MQ2E

\begin{tabular}{|c|c|c|c|c|}
\hline & (6) & (7) & (8) & (9) \\
\hline \multicolumn{5}{|c|}{ Variável dependente : $D K_{p c}=\log _{e}\left[K_{p c(2000)} / K_{p c(1991)}\right]$} \\
\hline \multirow[t]{2}{*}{ Const. } & -6.4976 & -1.4837 & -1.8021 & -2.8394 \\
\hline & $(0.3794)^{* * *}$ & $(0.7447)^{* *}$ & $(0.7420)^{* *}$ & $(1.0127)^{* * *}$ \\
\hline $\log K_{p c}$ & -0.5094 & -0.4093 & -0.4186 & -0.4236 \\
\hline$(1991)^{p c}$ & $(0.0152)^{* * *}$ & $(0.0202)^{* * *}$ & $(0.0203)^{* * *}$ & $(0.0255)^{* * *}$ \\
\hline $\log H_{p c}$ & 1.1106 & 0.4337 & 0.4844 & 0.6913 \\
\hline$(1991)^{p h}$ & $(0.0493)^{* * *}$ & $(0.0999)^{* * *}$ & $(0.0998)^{* * *}$ & $(0.1322)^{* * *}$ \\
\hline IQIM & & 0.2824 & 0.2751 & -0.0425 \\
\hline (2000) & & $(0.0368)^{* * *}$ & $(0.0363)^{* * *}$ & $(0.0854)$ \\
\hline Theil & & & -0.1690 & -0.1331 \\
\hline (1991) & & & $(0.0311)^{* * *}$ & $(0.0315)^{* * *}$ \\
\hline $\begin{array}{l}\text { Dum. Est. } \\
\text { (valor-p) }\end{array}$ & & & & $(0.00 \mathrm{e}+00)^{* * *}$ \\
\hline $\mathrm{R}^{2}$ & 0.399 & 0.375 & 0.382 & 0.529 \\
\hline $\mathrm{R}^{2}$ ajust & 0.399 & 0.374 & 0.382 & 0.526 \\
\hline $\mathrm{n}$ & 4491 & 4489 & 4489 & 4489 \\
\hline
\end{tabular}

NOTA: Erros-padrão robustos; ${ }^{* * *}$ significativo a $1 \%$; ** significativo a $5 \%$.

No modelo (6), a magnitude do capital humano quase dobra em relação a (1). Em (7) e (8), a inclusão das instituições reduz demasiadamente o coeficiente de $H_{p c}$ IQIM aumenta seu valor substancialmente em relação às estimações MQO. O índice de Theil permanece com valor próximo à sua contraparte da Tabela 1. O último modelo mostra que efeitos específicos

19 As variáveis geográficas são: (1) latitude; (2) precipitação pluviométrica (média, mm/mês); (3) temperatura média anual $\left({ }^{\circ} \mathrm{C}\right)$; e (4) altitude (metros). 
dos municípios podem estar correlacionados com os instrumentos, trazendo insignificância estatística para o coeficiente das microinstituições, em (9).

\section{CONCLUSÃO}

O presente artigo procurou determinar as variáveis importantes para acumulação do capital físico nos municípios brasileiros entre os anos de 1991 e 2000. As desigualdades no investimento líquido entre as municipalidades foram explicadas a partir dos modelos de crescimento endógeno e da teoria institucionalista, isto é, da literatura de longo prazo.

Os resultados dão indícios favoráveis às teorias de capital humano e instituições: mesmo após o controle da endogeneidade através do MQ2E - utilizando variáveis geográficas como instrumentos para o capital humano e instituições - as duas permaneceram com significância estatística em praticamente todas as especificações.

\section{REFERÊNCIAS}

ACEMOGLU, D.; JOHNSON, S.; ROBINSON, J. The colonial origins of comparative development: an empirical investigation. The American Economic Review, v. 91, n. 5, p. 13691401, 2001.

ACEMOGLU, D.; JOHNSON, S.; ROBINSON, J. Reversal of fortune: geography and institutions in the making of the modern world income distribution. The Quarterly Journal of Economics, p. 1231-1294, 2002.

ACEMOGLU, D.; JOHNSON, S.; ROBINSON, J.; YUNYONG, T. Institutional causes, macroeconomic symptoms: volatility, crises and growth. Journal of Monetary Economics, v. 50, p. 49-123, 2003.

ALVES, J.; LUPORINI, V. Evolução da teoria do investimento e análise empírica para o Brasil. In: XXXV ENCONTRO NACIONAL DE ECONOMIA. Disponível em: < http://www. anpec.org.br/encontro2007/artigos/A07A172.pdf>.

BARRO, R. Economic growth in a cross section of countries. The Quarterly Journal of Economics, v. 106, n. 2, p. 407-443, 1991.

BENHABIB, J.; SPIEGEL, M. The role of human capital in economic development: evidence from aggregate cross-country data. Journal of Monetary Economics, v. 34, p. 143-173, 1994.

EASTERLY, W.; LEVINE, R. It's not factor accumulation: stylized facts and growth models. The World Bank Economic Review, v. 15, p. 177-219, 2001.

EASTERLY, W.; LEVINE, R. Tropics, germs, and crops: how endowments influence economic development. Cambridge: National Bureau of Economic Research, 2002.

ENGERMAN, S.; SOKOLOFF, K. Factor endowments, inequality, and paths of development among new world economies. Economia, v. 3, n. 1, p. 41-88, 2002.

GLAESER, E.; LA PORTA, R.; LOPEZ-DE-SILANES, F.; SHLEIFER, A. Do institutions cause growth? Journal of Economic Growth, v. 9, p. 271-303, 2004. 
HALL, R.; JONES, C. I. Why do some countries produce so much more output per worker than others? The Quarterly Journal of Economics, v. 114, n. 1, p. 83-116, 1999.

JONES, C. I. Time series tests of endogenous growth models. Quarterly Journal of Economics, v. 110, n. 2, p. 495-525, 1995.

JONES, C. I. R\&D-Based models of economic growth. The Journal of Political Economy, v. 103, n. 4, p. 759-784, 1995.

JORGENSON, D. Capital theory and investment behavior. The American Economic Review, v. 53, n. 2, p. 247-259, 1963.

LUCAS, R. On the mechanics of economic development. Journal of Monetary Economics, v. 22, p. 3-42, 1988.

LUCAS, R. Why doesn't capital flow from rich to poor countries? American Economic Review, v. 80, p. 92-96, 1990.

MELO, G. M.; RODRIGUES JÚNIOR, W. Determinantes do investimento privado no Brasil: 1970-1995. Texto para discussão IPEA, n. 605, 1998.

NAKABASHI, L.; FIGUEIREDO, L. Mensurando os impactos diretos e indiretos do capital humano sobre o crescimento. Economia Aplicada, v. 12, n. 1, p. 151-171, 2008.

NARITOMI, J. Herança colonial, instituições e desenvolvimento. Dissertação (Mestrado) - Programa de Pós-Graduação em Economia, Pontifícia Universidade Católica do Rio de Janeiro, Rio de Janeiro, 2007.

NELSON, R.; PHELPS, E. Investment in humans, technological diffusion, and economic growth. The American Economic Review, v. 56, n. 1, p. 69-75, 1966.

PEREIRA, A. E. G.; NAKABASHI, L.; SACHSIDA, A. Qualidade das instituições e PIB per capita nos municípios brasileiros. Economia \& Tecnologia, Curitiba, 2010. Discussão.

ROMER, P. Endogenous technological chance. The Journal of Political Economy, v. 98, n. 5(2), p. S71-S102, 1990.

SALA-I-MARTIN, X. Fifteen years of new growth economics: what have we learned? In: LOYAZA, N., SOTO, R. Economic growth: sources, trends and cycles. Central Bank of Chile, 2002.

SONAGLIO, C.; BRAGA, M.; CAMPOS, A. Investimento público e privado no Brasil: evidências dos efeitos crowding-in e crowding-out no período 1995-2006. Economia, Brasília, v. 11, n. 2, p. 383-401, 2010.

SOLOW, R. Technical change and the aggregate production function. The Review of Economics and Statistics, v. 39, p. 312-320, 1957. 